The AstrophysicAl JouRnAL, 561:496-503, 2001 November 10

(C) 2001. The American Astronomical Society. All rights reserved. Printed in U.S.A.

\title{
SUPPRESSION OF GRAVITATIONAL STRUCTURE FORMATION BY COSMOLOGICAL ACCRETION HEATING
}

\author{
M. Coleman Miller and Eve C. Ostriker \\ Department of Astronomy, University of Maryland, College Park, MD 20742-2421; miller@astro.umd.edu, ostriker@astro.umd.edu \\ Received 2001 May 28; accepted 2001 July 12
}

\begin{abstract}
As increasingly precise information about the spectrum of the cosmic microwave background fluctuations is gathered with balloon and satellite experiments, interest has grown in foreground sources of opacity affecting these observations. One potentially important source is electron scattering produced by postrecombination luminosity, which would significantly attenuate the higher harmonics in the spectrum. If such an ionization source exists, then it would also heat the universe, hence increasing the Jeans mass and suppressing early gravitational structure formation. Here we consider the effects of such heating. We concentrate on one type of ionization source: luminosity generated by accretion onto primordial compact objects. We show that if such objects generate enough luminosity to affect the cosmic microwave background power spectrum, then they would produce enough heat to prevent the formation of 1 $\sigma$ collapsed objects until $z \sim 5$, significantly less than the redshift at which baryonic collapse could otherwise occur. Such processes would leave signatures detectable by upcoming instruments such as the Next Generation Space Telescope, the SIRTF, and Swift.

Subject headings: accretion, accretion disks — cosmic microwave background - cosmology: theory galaxies: formation
\end{abstract}

\section{INTRODUCTION}

The advent of a data-rich era in cosmology is expected to produce a dramatically enhanced understanding of the early universe, from the initial spectrum of perturbations and their processing before radiation decoupling to the formation of the first galaxies and stars. The high angular resolution power spectra of the cosmic microwave background (CMB) gathered by current and future satellite, balloon, and ground-based experiments are expected to provide precise information about many cosmological parameters, such as the total curvature of the universe and the baryonic mass fraction. These power spectra are also expected to be informative about a number of foreground sources, such as clusters of galaxies and other large-scale structure, and about the epoch of ionization of the intergalactic medium (e.g., Tegmark et al. 2000).

Early results from BOOMERANG and MAXIMA data suggested, surprisingly, that the second acoustic peak is significantly weaker than expected (de Bernardis et al. 2000; Hanany et al. 2000). More recent results from BOOMERANG (Netterfield et al. 2001), the Degree Angular Scale Interferometer (DASI; Pryke et al. 2001), and possibly MAXIMA (Lee et al. 2001) are consistent with the standard $\Lambda$ cold dark matter (CDM) cosmology. However, the systematic uncertainties in current data are significant enough that it is still not possible to tell with precision whether the higher harmonics are at the expected amplitude; this will need to wait for instruments with absolute calibration, such as the Microwave Anisotropy Probe (MAP) and the Planck satellite (e.g., Tegmark et al. 2000). Therefore, despite the encouraging agreement with expectations, it is worthwhile to calculate the impact that other effects have at both high and low redshifts.

The CMB spectrum can strongly constrain ionization at high redshifts, but some ionizing processes that may have only subtle effects (that are nonetheless measurable with $M A P$ and Planck) at $z \sim 1000$ can have important consequences at lower redshifts. One such effect within the stan- dard model has been suggested recently by Peebles, Seager, $\& \mathrm{Hu}(2000)$ : if there exists a radiation source in the universe around the time of decoupling $(z \sim 1000)$, the resulting enhanced density of free electrons at high redshift would Thomson-scatter the CMB and suppress the second and higher peaks, in addition to moving the first acoustic peak to larger angular scales. Such sources could have many additional cosmological effects. In particular, if scattering is posited to suppress the second acoustic peak (Peebles et al. 2000), then the luminosity responsible for increased ionization would also heat the universe. As a result, the Jeans mass at early epochs would be raised, which in turn could affect the redshift of collapse of the first nonlinear structures.

One candidate for an early ionization source is accreting compact objects that exist by a redshift $z \sim 1000$, e.g., black holes formed during the quark-hadron phase transition. The first detailed investigation of the effects of such objects was performed by Carr (1981). He showed that the radiation generated by accretion of ambient gas onto primordial black holes could have a significant effect on the thermal and ionization history of the universe, potentially even preventing the universe from entering a neutral phase. Given large-amplitude baryonic fluctuations, massive black holes could have formed soon after recombination; Gnedin \& Ostriker (1992) and Gnedin, Ostriker, \& Rees (1995) considered the possible consequences for thermal, ionization, and nucleosynthesis histories of early injection of an active galactic nucleus-like spectrum from such accreting sources.

An update to Carr's analysis was performed by Miller (2000), who used the existing limits on electron scattering optical depth between decoupling and the current epoch to place an upper bound on the contribution of primordial compact objects to dark matter. This limit is based on the effect such scattering would have on the angular power spectrum (limits based on distortions of the energy spectrum are much weaker; see, e.g., Griffiths, Barbosa, \& Liddle 1999). Miller found that the product $\Omega_{\mathrm{CO}} \epsilon_{-1}(M / 10$ 
$\left.M_{\odot}\right)<4 \times 10^{-3}$ (in the notation of the current paper, $\xi<4 \times 10^{-3}$ ), where $\Omega_{\mathrm{CO}}$ is the fraction of closure density in compact objects, $\epsilon_{-1}$ is the accretion efficiency divided by $10 \%$, and $M$ is the typical mass of the compact object. In this paper we show that current data imply $\xi \lesssim 5 \times 10^{-4}$, which corresponds to a limit on optical depth that is 3 times stronger than that used by Miller (2000) based on earlier data.

Here we explore the effect of ionizing sources on the gravitational structure formation history of the universe. We show that if accreting primordial compact objects generate enough ionization to significantly affect the second and third acoustic CMB peaks, then they raise the temperature of the universe to $T \gtrsim 500 \mathrm{~K}$ when $z \lesssim 30$. As has been discussed by a number of authors (e.g., Ostriker \& Gnedin 1996; Valageas \& Silk 1999; Haiman, Abel, \& Rees 2000; Gnedin 2000), an increase in temperature can dramatically alter the progress of structure formation. We show that in the present scenario, the Jeans instability threshold redshift for an $n-\sigma$ density fluctuation is reduced by $\sim 5-7$, compared to the threshold redshift when no extra heating sources are present. For example, $1-\sigma$ fluctuations can collapse only at $z \approx 5$.

We find that the parameter $\xi=\Omega_{\mathrm{CO}} \epsilon_{-1}\left(M / 10 M_{\odot}\right)$ governs both the distorting effect on the CMB power spectrum and the formation of large-scale structure. Because the two effects are therefore linked, we do not need to assume anything about the accretion efficiency $\epsilon$ for the compact objects; instead, we may simply say that if accretion onto compact objects has a given effect on the CMB power spectrum, it will have a related (and much stronger) effect on the redshift at which nonlinear density peaks may form in baryons. If the accretion efficiency is low (e.g., if the flow is governed by an advection-dominated, convectiondominated, or wind-dominated solution, for which $\epsilon$ may be less than $10^{-4}$; see, e.g., Narayan, Mahadevan, \& Quataert 1998; Ball, Narayan, \& Quataert 2001; Blandford \& Begelman 1999), all effects are proportionally reduced, but what we emphasize here is the relation between possible subtle effects at high redshift and significant effects at lower redshift.

In $\S 2$ we calculate the ionization fraction produced by an accreting compact object beyond the immediate vicinity of the compact object ("H II region") in the surrounding ambient region, which contains many such objects. In order to allow for nonlinear mass concentrations, we generalize the analysis of Miller (2000) to include accretion in a region with density differing from the average density of the universe. In $\S 3$ we compute the temperature of the $\mathrm{H}$ II and ambient regions by balancing accretion heating with Compton cooling off the CMB and also assesses the importance of bremsstrahlung, atomic cooling, and molecular cooling. In $\S 4$ we determine the heating implied if Thomson scattering is responsible for reducing the second peak to presently observed levels. We then evaluate the effect this level of heating would have on the formation of selfgravitating structures. In $\S 5$, we discuss the effects of more general ionization and heating and present our conclusions.

\section{IONIZATION FRACTION}

In this section we focus on accreting primordial compact objects as the source of early ionization and heating. In $\S 5$ we revisit the heating effects of more general early ioniza- tion sources. The physical picture is of compact objects of typical mass $M$ capturing matter via Bondi-Hoyle accretion from a surrounding medium. For generality we consider a medium with an ambient hydrogen number density $n_{\text {amb }}$ and temperature $T_{\mathrm{amb}}$ that may differ from the average density $\bar{n}$ and temperature $\bar{T}$ of the universe. This will allow us to treat accretion in underdense or overdense mass concentrations. The luminosity generated by accretion will produce an $\mathrm{H}$ II region immediately around each compact object, in which the ionization fraction $x \approx 1$. Let the number density and temperature inside the $\mathrm{H}$ II region be $n_{\mathrm{H} \mathrm{II}}$ and $T_{\mathrm{H} \mathrm{II}}$, respectively. As discussed by many authors (e.g., Silk 1971; Carr 1981; Miller 2000), the hardness of the spectrum produced by an accreting compact object implies that its ionization effect is felt over much greater distances than the radius of its Strömgren sphere and that the resulting ionization fraction decreases much more slowly with distance than is typical around an early-type star (as a power law, not exponentially).

Miller (2000) calculated the average ionization fraction for a differential luminosity spectrum typical of accreting black holes in our Galaxy, $d L(E) / d E \propto E^{-1} \exp \left(-E / E_{\max }\right)$ (the range of spectra observed from stellar-mass black holes all give similar answers for the ionization fraction; see Miller 2000 for a more detailed discussion). Typically, $E_{\max } \sim 100 \mathrm{keV}$. Over a region large compared with the radius of the Strömgren sphere, the average ionization fraction at redshifts $z \lesssim 100$ is

$$
\bar{x} \approx 3\left[\frac{E_{\max }}{3 E_{0} \ln \left(E_{\max } / E_{0}\right)}\right]^{1 / 2}\left(\frac{R_{\mathrm{S}}}{R_{\mathrm{sep}}}\right)^{3 / 2} .
$$

Here $E_{0}=13.6 \mathrm{eV}$ is the ground-state ionization energy of hydrogen, and the number of compact objects per cosmological volume is $\left(4 \pi R_{\mathrm{sep}}^{3} / 3\right)^{-1}$. In terms of the fraction $\Omega_{\mathrm{CO}}$ of closure density in compact objects and the redshift $z$,

$$
\begin{aligned}
R_{\text {sep }}= & 8 \times 10^{20} \mathrm{~cm}(1+z)^{-1} \\
& \times\left(\frac{M}{10 M_{\odot}}\right)^{1 / 3}\left(\frac{n_{\mathrm{amb}}}{\bar{n}}\right)^{-1 / 3} \Omega_{\mathrm{CO}}^{-1 / 3},
\end{aligned}
$$

where we adopt $H_{0}=70 \mathrm{~km} \mathrm{~s}^{-1} \mathrm{Mpc}^{-1}$. The Strömgren radius is here defined as

$$
R_{\mathrm{S}} \equiv\left(\frac{2 L}{3 \pi \alpha n_{\mathrm{amb}}^{2} E_{\mathrm{max}}}\right)^{1 / 3} .
$$

Here $\alpha=1.5 \times 10^{-12} T_{3}^{-0.75} \mathrm{~cm}^{3} \mathrm{~s}^{-1}$ is the recombination coefficient to the $n \geq 2$ states of hydrogen (Hummer 1994); recombination to the $n=1$ state produces photons that are absorbed almost immediately, taking the standard "case B" assumption. We scale the temperature as $T=10^{3} T_{3} \mathrm{~K}$ because we find typical temperatures $T \sim 10^{3} \mathrm{~K}$ at the redshifts of interest. This form for the recombination coefficient is accurate to better than $10 \%$ for all $T>10^{2} \mathrm{~K}$ and to $\sim 1 \%-2 \%$ for $10^{3} \mathrm{~K}<T<10^{5} \mathrm{~K}$ (Hummer 1994). The Bondi-Hoyle accretion rate is $\dot{M}=4 \pi \lambda_{s}(G M)^{2} \rho c_{s}^{-3}$, where for a $\gamma=5 / 3$ gas, the accretion eigenvalue is $\lambda_{s}=0.25$. This produces a luminosity per source $L$ given by

$$
\begin{aligned}
L \approx & 3 \times 10^{27} \mathrm{ergs} \mathrm{s}^{-1} \epsilon_{-1}(1+z)^{3} \\
& \times\left(\frac{M}{10 M_{\odot}}\right)^{2} \frac{n_{\mathrm{H} \mathrm{II}}}{\bar{n}} T_{\mathrm{H} \mathrm{I}, 3}^{-3 / 2},
\end{aligned}
$$


adopting a cosmological baryon density $\bar{\rho}=3.6 \times 10^{-31} \mathrm{~g}$ $\mathrm{cm}^{-3}(1+z)^{3}$ from Tytler et al. (2000). The accretion efficiency is defined as $L \equiv 0.1 \epsilon_{-1} \dot{M} c^{2}$. The Strömgren radius is then

$$
\begin{aligned}
R_{\mathrm{S}} \approx & 4.4 \times 10^{19} \mathrm{~cm}(1+z)^{-1} \epsilon_{-1}^{1 / 3}\left(\frac{E_{\max }}{10^{4} E_{0}}\right)^{-1 / 3} \\
& \times\left(\frac{M}{10 M_{\odot}}\right)^{2 / 3}\left(\frac{n_{\mathrm{H} \mathrm{II}}}{\bar{n}}\right)^{1 / 3}\left(\frac{n_{\mathrm{amb}}}{\bar{n}}\right)^{-2 / 3} T_{\mathrm{H} \mathrm{II}, 3}^{-1 / 2} T_{\mathrm{amb}, 3}^{1 / 4} .
\end{aligned}
$$

Pressure balance between the ambient medium and the $\mathrm{H}$ II region implies $n_{\mathrm{H} \mathrm{II}} T_{\mathrm{H} \mathrm{II}}=n_{\text {amb }} T_{\text {amb }}$. Collecting factors and defining $\xi \equiv \epsilon_{-1}\left(M / 10 M_{\odot}\right) \Omega_{\mathrm{CO}}$ as a parameter quantifying the generation of luminosity by accretion, the average ionization fraction in the ambient medium for $z \lesssim 100$ is

$$
\bar{x} \approx 0.7 \xi^{1 / 2}\left[\frac{9}{\ln \left(E_{\max } / E_{0}\right)}\right]^{1 / 2}\left(\frac{T_{\mathrm{amb}}}{T_{\mathrm{H} \mathrm{I}}}\right)^{7 / 8} T_{\mathrm{H} \mathrm{\textrm {I } , 3}}^{-3 / 8} .
$$

The factor in brackets depends only on the spectrum and is very close to unity. We note that the result from equations (1) and (6) is a factor $\left[8 / \ln \left(E_{\max } / E_{0}\right)\right]^{1 / 2} \sim 1$ times the result obtained by equating the total ionization at a mean rate $n_{\mathrm{CO}} L /\left(3 E_{0}\right)$ with recombinations at a mean rate $\alpha n_{\mathrm{amb}}^{2} x^{2}$. Because $L \propto n$ for energy generated via cosmic accretion, the resulting mean cosmic ionization is density independent except indirectly through the temperature.

\section{BALANCE OF HEATING AND COOLING}

The formation of baryonic structure depends in part on whether the nonlinear mass at a given redshift exceeds the Jeans mass at that epoch. In $\S 4$ we will derive the nonlinear mass. The Jeans mass depends on the typical temperature of the regions that may undergo collapse. We therefore need to compute this temperature in the presence of accretion heating and ionization.

The temperature $T_{\text {amb }}$ of the ambient medium is determined by the balance of heating by accretion with cooling by a variety of processes. Since ionization is not perfectly efficient (i.e., only a fraction of available energy goes into ionization), heating by accretion is inevitable. Cooling depends on a number of processes: candidates include Compton cooling, bremsstrahlung, atomic recombination, and molecular cooling. In addition to considering ionization and thermal balance in the ambient medium, we must also evaluate the latter in the $\mathrm{H}$ II region. This is important because, at least for heating by accretion, the luminosity per compact object depends on both the temperature and the density inside the $\mathrm{H}$ II region (the Bondi radius is smaller than $R_{\mathrm{S}}$ for $\left.M \lesssim 10^{4} M_{\odot}\right)$. We first compute the $\mathrm{H}$ in region and ambient temperatures $T_{\mathrm{H}}, T_{\text {amb }}$ by balancing accretion heating and Compton cooling in each region separately, assuming that pressure balance applies at the interface. We then demonstrate self-consistency by showing that if ionization is dominated by radiative ionization (as opposed to collisional ionization), then cooling by bremsstrahlung, atomic recombination, and the formation of $\mathrm{H}_{2}$ can be ignored. Only Compton cooling is effective.

Balance of accretion heating and Compton cooling.--In general, the heating rate is related to the ionization rate, with the heating rate per unit volume $\Gamma=\mathscr{E}_{T} \zeta(1-x) n_{\mathrm{H}}$ for $\mathscr{E}_{T}$, the typical excess (thermal) energy in each ionization, and $\zeta$, the ionization coefficient. As discussed by Carr
(1981), through ionization balance the heating rate may be related to the recombination rate as $\Gamma=\mathscr{E}_{T} \alpha \times n_{\mathrm{H}} n_{e}$. Numerically, the heating rate at $z \lesssim 100$ is thus

$$
\Gamma \approx 6.2 \times 10^{-23} \mathrm{ergs} \mathrm{cm}^{-3} \mathrm{~s}^{-1} x n_{\mathrm{H}} n_{e} \frac{\mathscr{E}_{T}}{2 E_{0}} T_{3}^{-0.75},
$$

where the number densities here (and everywhere else in this paper) are measured in units of $\mathrm{cm}^{-3}$. Carr (1981) estimates that there is $\approx 7 \mathrm{eV}$ of heating per ionization within the $\mathrm{H}$ II region, in which primarily soft photons interact because of their higher absorption cross sections. In the ambient medium far from the source, the soft photons have already been absorbed, and hence the photons are harder. The number of ionizations per primary of energy $E$ is then $\sim E / 3 E_{0}$ for typical photon energies $\sim 1 \mathrm{keV}$ or higher (Dalgarno, Yan, \& Liu 1999), so that $\mathscr{E}_{T} \sim E\left(E / 3 E_{0}\right)^{-1}$ $-E_{0}=2 E_{0}$.

After decoupling but prior to structure formation, the entire universe is nearly transparent to CMB photons. Scattering of these photons off free electrons provides a volume cooling rate $4 \sigma_{\mathrm{T}} a T_{\mathrm{CMB}}^{4} k\left(T-T_{\mathrm{CMB}}\right) n_{e} /\left(m_{e} c\right)$, (where $\sigma_{\mathrm{T}}$ is the Thomson scattering cross section), or

$$
\Lambda_{\mathrm{CMB}}=5.6 \times 10^{-33} \mathrm{ergs} \mathrm{cm}^{-3} \mathrm{~s}^{-1} n_{e}(1+z)^{4}\left(T_{3}-T_{\mathrm{CMB}, 3}\right) .
$$

At the redshifts $z \ll 1000$ relevant to our heating calculation, the temperature in the $\mathrm{H}$ II region or ambient medium is much greater than the temperature of the CMB, and hence the factor in parentheses is approximately $T_{3}$. Inside the $\mathrm{H}$ II region, we set $n_{\mathrm{H}}$ and $n_{e}$ to $n_{\mathrm{H}}, x \approx 1$ and $T$ to $T_{\mathrm{H} \text { II }}$; in the ambient region we set $n_{\mathrm{H}}$ to $n_{\mathrm{amb}}, n_{e}$ to $x n_{\mathrm{amb}}$, and $T$ to $T_{\text {amb }}$ and evaluate $x$ from equation (6). From pressure balance with the surrounding ambient medium, $n_{\mathrm{H} \mathrm{II}} T_{\mathrm{H} \text { II }}=n_{\text {amb }} T_{\text {amb }}$.

Equating the accretion heating and CMB Compton cooling rates (eqs. [7] and [8]) in the $\mathrm{H}$ II region, we find

$$
T_{\mathrm{H} \mathrm{II}, 3}=10(1+z)^{-4 / 11} T_{\mathrm{amb}, 3}^{4 / 11}\left(\frac{n_{\mathrm{amb}}}{\bar{n}}\right)^{4 / 11}\left(\frac{\mathscr{E}_{T, \mathrm{H} \mathrm{II}}}{2 E_{0}}\right)^{4 / 11} .
$$

Similarly, thermal balance in the ambient region yields

$$
T_{\mathrm{amb}, 3}=70(1+z)^{-4 / 7} \bar{x}^{4 / 7}\left(\frac{n_{\mathrm{amb}}}{\bar{n}}\right)^{4 / 7}\left(\frac{\mathscr{E}_{T, \mathrm{amb}}}{2 E_{0}}\right)^{4 / 7} .
$$

Combining this with our expressions from equation (6) for the ionization fraction $\bar{x}$ in the ambient medium and equation (9) for the temperature of the $\mathrm{H}$ II region, we solve to obtain

$$
\begin{aligned}
T_{\mathrm{amb}, 3}= & 28(1+z)^{-2 / 5} \xi^{5 / 13}\left(\frac{n_{\mathrm{amb}}}{\bar{n}}\right)^{2 / 5}\left(\frac{\mathscr{E}_{T}}{2 E_{0}}\right)^{2 / 5} \\
& \times\left[\frac{9}{\ln \left(E_{\mathrm{max}} / E_{0}\right)}\right]^{5 / 13}, \\
T_{\mathrm{H} \mathrm{II}, 3}= & 33(1+z)^{-1 / 2} \xi^{1 / 7}\left(\frac{n_{\mathrm{amb}}}{\bar{n}}\right)^{1 / 2}\left(\frac{\mathscr{E}_{T}}{2 E_{0}}\right)^{1 / 2} \\
& \times\left[\frac{9}{\ln \left(E_{\max } / E_{0}\right)}\right]^{1 / 7},
\end{aligned}
$$


and

$$
\begin{aligned}
\bar{x}= & 0.19(1+z)^{3 / 11} \xi^{2 / 3}\left(\frac{n_{\mathrm{amb}}}{\bar{n}}\right)^{-3 / 11}\left(\frac{\mathscr{E}_{T}}{2 E_{0}}\right)^{-3 / 11} \\
& \times\left[\frac{9}{\ln \left(E_{\max } / E_{0}\right)}\right]^{2 / 3} .
\end{aligned}
$$

The exponents are not exact but are close approximations to the formally derived exponents. We neglect possible differences between $\mathscr{E}_{T}$ in the $\mathrm{H}$ II and ambient regions. Equations (11)-(13) apply at redshifts where $T \gg T_{\mathrm{CMB}}$; as $z$ approaches decoupling, the effects of accretion become negligible. Figure 1 shows the high-redshift ambient ionization boost for $\xi=5 \times 10^{-4}$ (which we find in $\S 4.1$ gives a good fit to the second-year BOOMERANG and DASI data), compared with the standard model in which there is no extra ionization and hence $\xi=0$.

We now consider three other cooling mechanisms: atomic recombination, bremsstrahlung, and molecular cooling by the formation of $\mathrm{H}_{2}$. For each of these we assume that the ionization fraction $x$ is determined by the radiative ionization balance calculated earlier. In very dense or hot media, collisional ionization will raise the ionization fraction and therefore increase the effectiveness of these cooling mechanisms.

Atomic recombination.--In solving for the ionization fraction by equating the recombination rate with the radiative ionization rate, we have already effectively taken atomic recombination cooling into account. This is because the thermal energy, $k T<1 \mathrm{eV}$, is much less than the ionization energy of $13.6 \mathrm{eV}$. Therefore, recombination plays a minor

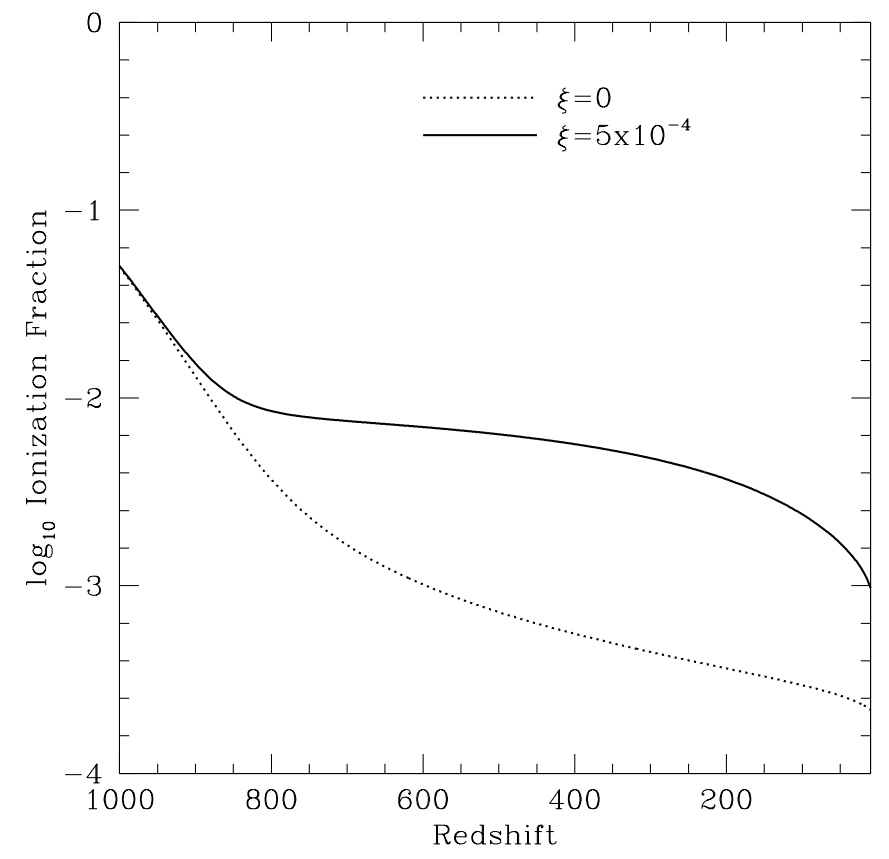

FIG. 1.-Fractional ionization $x$, as a function of redshift, for accretion by primordial compact objects $\left(\xi=5 \times 10^{-4}\right.$, the best fit to the BOOMERANG and DASI data; see $\S 4.1$ ) and for no extra ionization source $(\xi=0)$. Other cosmological parameters are chosen as in $\S 4.1$. The relatively small difference at high redshifts implies that effects on the CMB power spectrum are small (albeit measurable), but the large difference at lower redshift is one consequence of the significant heating effect such sources can have during the epoch of nonlinear structure formation. role in cooling the medium. If the temperature were high enough that collisional ionization were important, then recombination might play a major role, but here it can be neglected.

Bremsstrahlung.-The bremsstrahlung cooling rate is

$$
\Lambda_{\text {bremss }}=4.4 \times 10^{-26} \mathrm{ergs} \mathrm{cm}^{-3} \mathrm{~s}^{-1} x n_{\mathrm{H}} n_{e} T_{3}^{1 / 2} .
$$

The ratio of bremsstrahlung cooling to the heating rate is thus $\approx 6 \times 10^{-4} T_{3}^{5 / 4}$, which is small for the present situation.

Molecular cooling.- No metals are present in the early universe, so the only ways to form $\mathrm{H}_{2}$ (by far the dominant molecule) are the two-stage processes (1) $\mathrm{H}+e^{-} \rightarrow \mathrm{H}^{-}+\gamma$ followed by $\mathrm{H}^{-}+\mathrm{H} \rightarrow \mathrm{H}_{2}+e^{-}$and $(2) \mathrm{H}^{+}+\mathrm{H} \rightarrow \mathrm{H}_{2}^{+}$ $+\gamma$ followed by $\mathrm{H}_{2}^{+}+\mathrm{H} \rightarrow \mathrm{H}_{2}+\mathrm{H}^{+}$. Puy et al. (1993) give rates for these reactions, and the first process dominates by at least 2 orders of magnitude over the temperature range of interest. Moreover, the second step of process 1 is effectively instantaneous compared with the first, so the reaction rate for $\mathrm{H}+e^{-} \rightarrow \mathrm{H}^{-}+\gamma$ determines the overall rate of $\mathrm{H}_{2}$ formation. This rate is $\alpha_{\mathrm{mol}}=10^{-15} \mathrm{~s}^{-1} \mathrm{~cm}^{3} T_{3}$. Assuming as an upper bound that all $\sim 10 \mathrm{eV}$ released in this process escapes to infinity, the cooling rate in the (lowionization) ambient medium is

$$
\Lambda_{\mathrm{mol}}=10 \mathrm{eV} \times \alpha_{\mathrm{mol}} n_{e} n_{\mathrm{amb}} .
$$

The ratio of this molecular cooling to the mean accretion heating is $2.7 \times 10^{-4} x^{-1} T_{3}^{7 / 4}$. From equations (11) and (13), this ratio becomes

$$
\Lambda_{\mathrm{mol}} / \Gamma=0.5(1+z)^{-1} \frac{n_{\mathrm{amb}}}{\bar{n}} \frac{\mathscr{E}_{T, \mathrm{amb}}}{2 E_{0}} .
$$

This is generally less than unity for the redshift range of interest for regions that are not strongly overdense compared with the universe as a whole. Once a region becomes significantly condensed $\left(n_{\text {amb }} / \bar{n} \gg 1\right)$, molecular cooling begins to dominate other cooling terms (Lepp \& Shull 1984). For the purposes of assessing the onset of collapse, however, we will simply use the result expressed in equation (11), obtained in the approximation that accretion heating and CMB Compton cooling are in balance.

\section{EFFECTS ON THE FORMATION OF STRUCTURE}

With the ambient temperature $T_{\mathrm{amb}}$ that we derived in the previous section, we can calculate the Jeans mass within a region of ambient density $n_{\text {amb }}$ if we know the accretion parameter $\xi=\epsilon_{-1}\left(M / 10 M_{\odot}\right) \Omega_{\mathrm{CO}}$. We can then compare the Jeans mass as a function of redshift with the nonlinear mass scales at that redshift and hence evaluate, as a function of $\xi$, whether baryonic condensations within nonlinear dark matter peaks can collapse. In this section we first compute the maximum value of $\xi$ compatible with current $\mathrm{CMB}$ data, then derive the nonlinear masses, and determine the epoch of structure formation.

\subsection{Relation to the CMB Power Spectrum}

We use the code CMBFAST (version 4.0; Seljak \& Zaldarriaga 1996 and subsequent papers) to generate a power spectrum and compare it with the best current signal-tonoise ratio data, the second-year BOOMERANG (Netterfield et al. 2001) and DASI (Pryke et al. 2001) data. 
As an illustrative fit, we fixed all of the cosmological parameters except for $\xi$ and then fitted for $\xi$. We assume $\Omega_{b}=$ $0.05, \Omega_{\mathrm{CDM}}=0.29, \Omega_{\Lambda}=0.66$ (and hence assume a flat universe), $\Omega_{v}=0, H_{0}=66 \mathrm{~km} \mathrm{~s}^{-1} \mathrm{Mpc}^{-1}, T_{\mathrm{CMB}}(z=0)=$ $2.726 \mathrm{~K}, Y_{\mathrm{He}}=0.246$, and three generations of massless neutrinos and none of massive neutrinos. These numbers are consistent with fits of recent CMB data that include priors based on large-scale structure, Cepheid measurements of the Hubble constant, and Type Ia supernovae. This fit is also consistent with big bang nucleosynthesis. We also assume an $n=1$ initial perturbation spectrum and adiabatic initial conditions and do not include tensor perturbations. We included the additional ionization by modifying the routine recfast.f. At high redshift, the temperature of the $\mathrm{H}$ II region is no longer much greater than the temperature of the CMB, and hence for high $z$ we solve for the ionization numerically without using this assumption.

We plot the resulting curves for no accretion $(\xi=0)$, $\xi=5 \times 10^{-4}$ and $\xi=10^{-3}$, against the second-year BOOMERANG and DASI data in Figure 2. The vertical bars are the estimated statistical uncertainties in measurement. Netterfield et al. (2001) estimate that there is a $20 \%$ systematic uncertainty in the points, and Pryke et al. (2001) estimate that there is a $16 \%$ uncertainty in the power measurements, but for this figure we have plotted the raw output from CMBFAST against the data, including only statistical errors. Note that there are also substantial systematic errors contributed by beam uncertainties, particularly for $l>400$ (e.g., Netterfield et al. 2001), which have not been included in this figure. Clearly, the power spectrum is

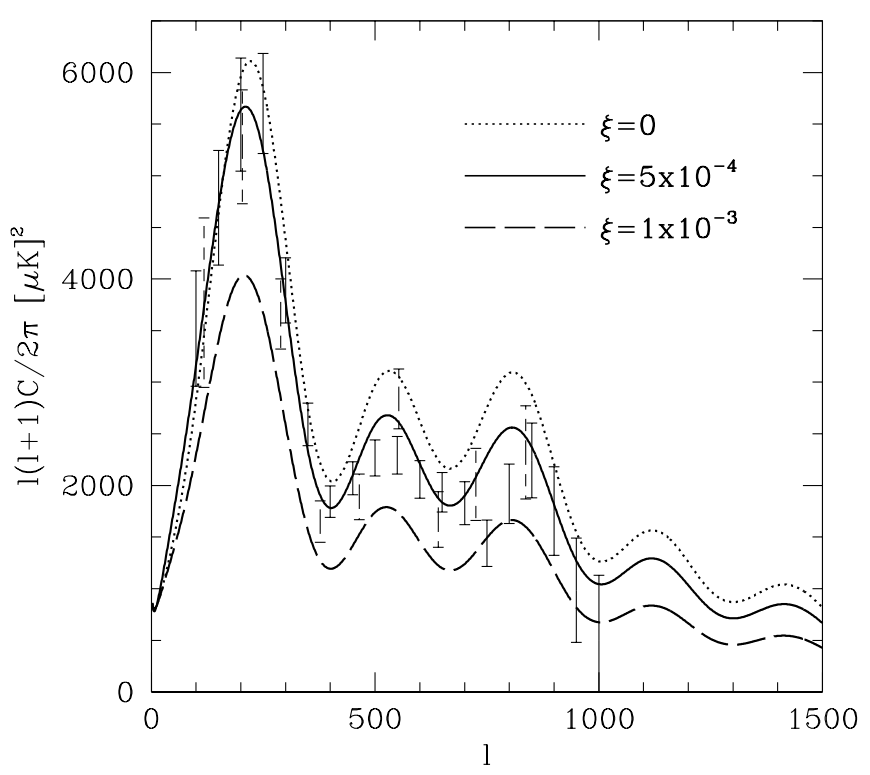

Fig. 2.-Comparison of CMBFAST output, using the compact object accretion model, with the second-year BOOMERANG data (Netterfield et al. 2001; solid error bars) and DASI data (Pryke et al. 2001; dashed error bars), including statistical but not systematic errors. Here only $\xi$ is varied, and other cosmological parameters such as $H_{0}$ and $\Omega_{\Lambda}$ are fixed at the values indicated in the text. When a $+20 \%$ shift is allowed, $\xi=0$ and $\xi=5 \times 10^{-4}$ fit well, but $\xi=10^{-3}$ still gives too low a first peak. Note that the amplitude of the second peak is quite sensitive to $\xi$. The best value, $\xi=5 \times 10^{-4}$, gives an acceptable fit: for example, compared with the BOOMERANG data, $\chi^{2}=26.2$ for 17 degrees of freedom, including an overall systematic shift of $5 \%$ but not including beam errors. fairly sensitive to $\xi$ in this range. To get a rough idea of the goodness of fit, we made a simplistic comparison of the model with the data at the center of each $l$ range, allowing an overall shift of up to $\pm 20 \%$ but not varying the data to accommodate beam uncertainties. The resulting $\chi^{2}$ values compared against the BOOMERANG data are 39.1 for $\xi=0,26.2$ for $\xi=5 \times 10^{-4}$, and 65.7 for $\xi=10^{-3}$. In each case there are 17 degrees of freedom, since there are 19 data points and $\xi$ and the overall scale are varied. The best value of $\xi=5 \times 10^{-4}$ gives a fit acceptable at the 7\% level, not including the beam errors, which make all of the curves significantly more compatible with the data. We note that the best-fit value of $\xi \equiv \epsilon_{-1} \Omega_{\mathrm{CO}}\left(M / 10 M_{\odot}\right)$ is compatible with a low value of the accretion efficiency $\epsilon$ and/or a low value of the fraction of closure mass in compact objects $\Omega_{\mathrm{CO}}$, provided the compact objects are relatively massive.

\subsection{Growth of Density Perturbations}

When density perturbations are linear, the relative amplitude $D(z)$ of a density perturbation $\delta=(\rho-\bar{\rho}) / \bar{\rho}$ evolves with the overall scale factor $a \equiv(1+z)^{-1}$ of the universe according to

$$
D(z)=E(z) \frac{5 \Omega_{m}}{2} \int_{z}^{\infty} \frac{1+z^{\prime}}{E\left(z^{\prime}\right)^{3}} d z^{\prime}
$$

(Heath 1977). Here $E(z)=H(t) / H_{0}=\left[\Omega_{m}(1+z)^{3}+\Omega_{r}\right.$ $\left.(1+z)^{4}+\Omega_{\Lambda}+\Omega_{k}(1+z)^{2}\right]^{1 / 2}, \Omega_{m}, \Omega_{r}$, and $\Omega_{\Lambda}$ denote the present contributions to $\Omega$ from matter, radiation, and the cosmological constant, and $\Omega_{k}=1-\left(\Omega_{m}+\Omega_{r}+\Omega_{\Lambda}\right)$ is the overall curvature (our naming convention corresponds to that of, e.g., Peebles 1993). For the regime of interest, we shall neglect the $\Omega_{r}$ term. The power spectrum of smallamplitude fluctuations at redshift $z$ thus evolves under linear growth according to

$$
P(k, z)=\left[\frac{D(z)}{D(0)}\right]^{2} P_{0}(k),
$$

where $k$ is the comoving wavenumber of a fluctuation.

To obtain the power spectrum shape and normalization, we follow the development of Bunn \& White (1997) and $\mathrm{Hu}$ $\&$ Sugiyama (1996). We assume that the power-law slope of the initial perturbations is $n=1$, that the universe is flat, $\Omega_{k}=0$, and that $k$, the comoving wavenumber, is measured in units of $\mathrm{Mpc}^{-1}$. Bunn \& White (1997) write the present-day power spectrum as $\Delta_{0}^{2}(k)=k^{3} P_{0}(k) / 2 \pi^{2}=$ $\delta_{\mathrm{H}}^{2}\left[k /\left(H_{0} / c\right)\right]^{3+n} T^{2}(k)$, with $\delta_{\mathrm{H}} \approx 2 \times 10^{-5} \Omega_{m}^{-0.73}$. The transfer function is given by (Bardeen et al. 1986)

$$
\begin{aligned}
T(q) & =T \frac{k}{h \Gamma}=\frac{\ln (1+2.34 q)}{2.34 q} \\
& {\left[1+3.89 q+(16.1 q)^{2}+(5.46 q)^{3}+(6.71 q)^{4}\right]^{-1 / 4} }
\end{aligned}
$$

where $h \Gamma \approx \Omega_{m} h^{2}$. From Carroll, Press, \& Turner (1992), a good approximation to the present growth factor is

$$
D(0) \approx \frac{5 \Omega_{m}}{2\left[\Omega_{m}^{4 / 7}-\Omega_{\Lambda}+\left(1+\Omega_{m} / 2\right)\left(1+\Omega_{\Lambda} / 70\right)\right]},
$$

so for $\Omega_{m}=0.3$ and $\Omega_{\Lambda}=0.7, D(0) \approx 0.8$.

The potential for collapsed objects of a given mass to form depends first of all on whether perturbations of the 
corresponding physical scale have grown sufficiently to become nonlinear. The variance in the fluctuation spectrum at a given mass $M$ (or comoving length scale $R$ ) is related to the power spectrum at redshift $z$ by the following equation (e.g., Barkana \& Loeb 2001):

$$
\sigma^{2}(M)=\sigma^{2}(R)=\int_{0}^{\infty} \frac{d k}{2 \pi^{2}} k^{2} P(k, z)\left[\frac{3 j_{1}(k R)}{k R}\right]^{2} ;
$$

we adopt a top-hat filter $j_{1}(x)=(\sin x-x \cos x) / x^{2}$.

A top-hat perturbation on comoving scale $R$ collapses in an Einstein-de Sitter universe at the point where its overdensity as predicted by linear theory reaches a value $\delta_{c}=$ 1.686 (e.g., Peebles 1993); this critical overdensity has only a weak dependence on cosmological parameters. The typical redshift for a $1 \sigma$ peak at mass scale $M$ to collapse is therefore given by the implicit solution of $\sigma(M)=\delta_{c}$ for $z$ using equation (21); the collapse redshift for a mass scale $M$ residing in a $2 \sigma$ peak is given by a solution of $\sigma(M)=\delta_{c} / 2$, and so on.

Whether the local baryons collapse into a given dark matter halo depends on the halo mass relative to the Jeans mass: baryons collapse with the halo only if the halo's gravitational potential $\left(\sim G M^{2 / 3} \rho^{1 / 3}\right)$ exceeds the specific thermal energy of baryons $(\sim k T / \mu)$. If we assume spherical density perturbations, then the Jeans mass for a cloud of temperature $T$ (and sound speed $c_{s}$ ) and total matter density $\rho_{\text {tot }}$ (including both baryons and CDM) is

$$
\begin{aligned}
M_{\mathrm{J}} & =\frac{1}{6} \pi \rho_{\mathrm{tot}}\left(\frac{\pi c_{s}^{2}}{G \rho_{\mathrm{tot}}}\right)^{3 / 2} \\
& \approx 7 \times 10^{5} M_{\odot}\left(\rho_{\mathrm{tot}} / \rho_{\mathrm{amb}}\right)^{-1 / 2} T_{\mathrm{amb}, 3}^{3 / 2} n_{\mathrm{amb}}^{-1 / 2}
\end{aligned}
$$

for a cosmological abundance of helium. Here $n_{\text {amb }}$ is measured in units of $\mathrm{cm}^{-3}$ and $\rho_{\mathrm{amb}}$ is the ambient density in baryons only. If we assume that during collapse the ratio of total mass to baryonic mass in a mass concentration remains approximately constant at the average value (roughly 9), then $\left(\rho_{\text {tot }} / \rho_{\text {amb }}\right)^{-1 / 2} \approx \frac{1}{3}$ and $M_{\mathrm{J}} \approx 2 \times 10^{5}$ $M_{\odot} T_{\mathrm{amb}, 3}^{3 / 2} n_{\mathrm{amb}}^{-1 / 2}$. In the standard model, in which there is no extra source of heating, the minimum halo mass for collapsed baryonic objects including the shell crossing by dark matter and other effects is (Barkana \& Loeb 2001)

$$
M_{\min }=5 \times 10^{3} M_{\odot}\left(\frac{1+z}{10}\right)^{3 / 2}\left(\frac{\Omega_{m} h^{2}}{0.15}\right)^{-1 / 2}\left(\frac{\Omega_{b} h^{2}}{0.022}\right)^{-3 / 5},
$$

which is almost identical to the value $M_{\mathrm{J} \text {,stand }}$ that the Jeans mass of equation (22) would take on in the absence of cosmic heating (see eq. [41] of Barkana \& Loeb 2001). If instead heating by accretion is included, then from the results of the previous section we obtain

$$
\begin{aligned}
M_{\mathrm{J}, \text { mod }} & \approx 8 \times 10^{10} M_{\odot}(1+z)^{-2} \xi^{7 / 12}\left(n_{\mathrm{amb}} / \bar{n}\right)^{1 / 10} \\
& \approx 8 \times 10^{8} M_{\odot} \xi^{7 / 12}[(1+z) / 10]^{-2} .
\end{aligned}
$$

Note that this mass is virtually independent of $n_{\mathrm{amb}}$. Since $\xi \sim 5 \times 10^{-4}$ fits the BOOMERANG data, this implies $M_{\mathrm{J}, \text { mod }} \approx 1.0 \times 10^{7} M_{\odot}[(1+z) / 10]^{-2}$. This mass is vastly greater than it would be sans heating and hence has a major effect on structure formation.

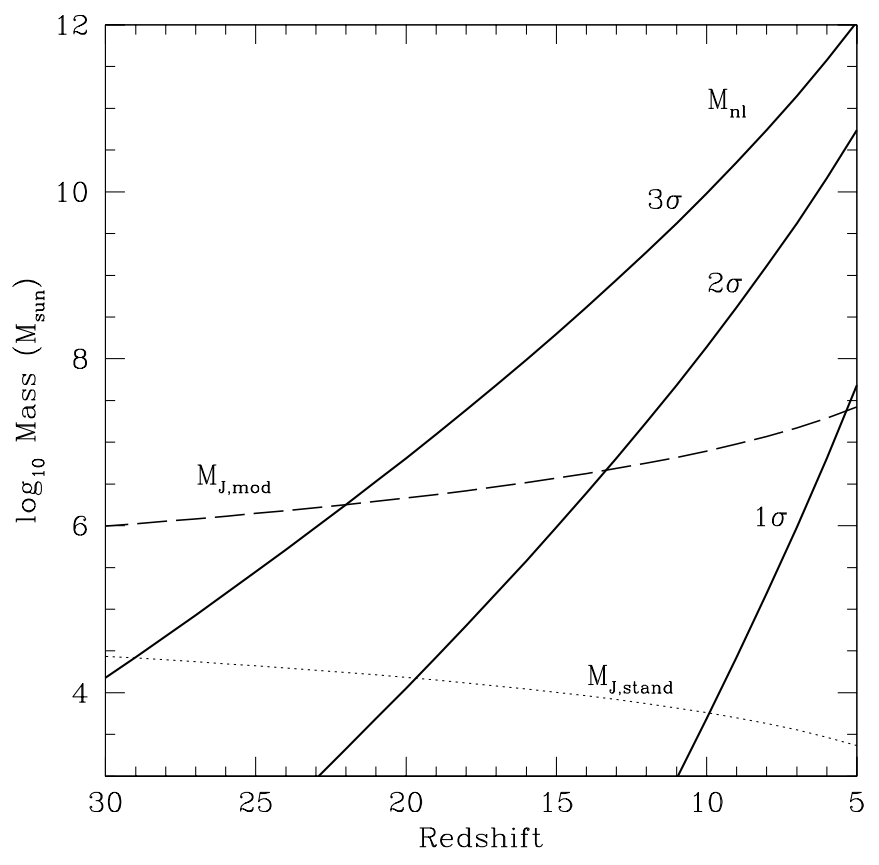

FIG. 3.-Nonlinear mass and Jeans mass as a function of redshift, with and without accretion heating. The solid lines show the nonlinear mass $M_{\mathrm{n} 1}$ for $1 \sigma, 2 \sigma$, and $3 \sigma$ peaks. The dotted line $\left(M_{\mathrm{J}, \text { stand }}\right)$ is the Jeans mass assuming no additional heating, and the long dashed line $\left(M_{\mathrm{J}, \bmod }\right)$ is the Jeans mass modified by heating $\left(\xi=5 \times 10^{-4}\right)$ compatible with the most recent BOOMERANG, MAXIMA, and DASI data. This figure shows that the effect of such heating is to delay baryonic structure formation drastically; typically the formation redshift of an $n \sigma$ peak is 5-7 less than what it would be without the heating.

Figure 3 exhibits this effect. Here we show, as a function of redshift, the nonlinear mass $M_{\mathrm{n} 1}$ for $1 \sigma, 2 \sigma$, and $3 \sigma$ peaks, along with the standard Jeans mass and the Jeans mass modified by accretion heating (with $\xi=5 \times 10^{-4}$ ). The nominal collapse redshift for a given rarity of peak is significantly less than it would be without heating.

\section{DISCUSSION AND CONCLUSIONS}

We have shown that if accretion onto primordial compact objects produces enough luminosity to increase significantly the ionization fraction of the universe at $z \sim 1000$, then this luminosity has a dominant effect on the thermal evolution of the universe at lower redshifts. In particular, the Jeans mass is raised by some 3 orders of magnitude at $z \sim 10$, which would substantially delay baryonic structure formation. Even if the ionization produced by compact objects is insufficient to affect the second CMB acoustic peak strongly, the moderate dependence of the Jeans mass in equation (24) on the accretion luminosity parameter $\xi$ implies that the presence of even a relatively small number of (evenly distributed) primordial compact objects could have a strong effect on structure formation.

Effects on the observed cosmic background radiation other than on the power spectrum are very subtle. For example, the existence of extra free electrons with a temperature elevated above the radiation temperature will distort the photon energy spectrum. To first order this can be characterized by the Compton $y$ parameter, $y=$ $\int d \tau k \Delta T / m_{e} c^{2}$. However, even with the enhanced ionization and temperature, $y=2 \times 10^{-9}$ when $\xi=5 \times 10^{-4}$, com- 
pared with the limit $|y|<7 \times 10^{-6}$ from $C O B E$ measurements (Mather et al. 1999). Thus, energy spectral distortions from scattering place far weaker limits on extra ionization than do limits to changes in the power spectrum (see also Griffiths et al. 1999). Another potential contributor to the energy spectrum is the luminosity from the accretion itself. However, this is also negligible. From equation (4), at $z \approx 10^{3}$ the luminosity per source is roughly $10^{36}$ ergs $\mathrm{s}^{-1} \epsilon_{-1}\left(M / 10 M_{\odot}\right)^{2}$. The total luminosity is $L \approx 2 \times 10^{57}$ ergs $\mathrm{s}^{-1} \epsilon_{-1} \Omega_{\mathrm{CO}}\left(M / 10 M_{\odot}\right)^{2}=2 \times 10^{57} \mathrm{ergs} \mathrm{s}^{-1} \xi(M / 10$ $\left.M_{\odot}\right)$. Taking redshifts into account and assuming a radius of $\approx 10^{28} \mathrm{~cm}$ for the universe, the observed flux would be $F \approx 2 \times 10^{-6} \xi$ ergs $\mathrm{cm}^{-2} \mathrm{~s}^{-1}$. Even for $\xi=1$ this is a factor of $\approx 10^{3}$ less than the flux from a $2.7 \mathrm{~K}$ blackbody. Taking into account that only a small fraction of the accretion radiation will be near the blackbody frequencies and that $\xi=5 \times 10^{-4}$, the ratio of fluxes is $\approx 10^{7}$. The direct radiation is unobservable. Thus, at high redshift the power spectrum places the strongest limits on accretion by primordial compact objects. However, as we have shown, at low redshifts the effects can be much more dramatic.

An important difference between ionization by accreting primordial objects and ionization by, e.g., the first generation of stars or quasars is that the latter only exist after ordinary structure has already formed, whereas the former ionize and heat the universe independent of any structure formation. Hence, accretion onto primordial objects, or any other mechanism independent of structure formation at moderate redshift, can in principle delay any structure formation. Similar effects from early stars or quasars are selflimiting and thus may not prevent - but nevertheless will regulate - further growth of structure (Ostriker \& Gnedin 1996).

More generally, any ionization source will also heat the universe. For example, Peebles et al. (2000) analyzed a simple model in which a uniform source of Ly $\alpha$ photons exists early in the universe. More hydrogen atoms are therefore in the $n=2$ state, so they can be ionized more easily. The enhanced ionization produces more free electrons, which scatter the CMB background, moving the surface of last scattering to lower redshift and therefore moving the first CMB peak to lower $l$ (larger angular scales) and decreasing the amplitude of the second CMB peak. In their model, they assume that the rate of production of $\mathrm{Ly} \alpha$ photons per unit volume is

$$
\frac{d n_{\alpha}}{d t}=\epsilon_{\alpha} n_{\mathrm{H}} H(t)
$$

Here $n_{\mathrm{H}}$ is the number density of hydrogen, $\epsilon_{\alpha}$ is a free parameter, and $H(t)=\dot{a} / a$ is the Hubble parameter at time $t$. Peebles et al. (2000) found that with this model, $\epsilon_{\alpha}=10$ gave a good fit to the first-year BOOMERANG data.

Let us assume that for each Ly $\alpha$ photon produced by the sources there is some heating as well and that the energy that heats the universe is a fraction $f$ of the energy in the Ly $\alpha$ photons themselves. At moderate and high redshifts with $\Omega_{m}=0.3$ and $H_{0}=70 \mathrm{~km} \mathrm{~s}^{-1} \mathrm{Mpc}^{-1}, H \approx 1.2 \times 10^{-18}$ $\mathrm{s}^{-1}(1+z)^{3 / 2}$; the baryon density is $n_{\mathrm{H}}=1.5 \times$ $10^{-7} \mathrm{~cm}^{-3}(1+z)^{3}$ from Tytler et al. (2000). The energy of a Ly $\alpha$ photon is approximately $10 \mathrm{eV}$, so the volume heating rate associated with equation (25) would be

$$
\begin{aligned}
\Gamma & =f \epsilon_{\alpha}(10 \mathrm{eV}) n_{\mathrm{H}} H(t) \\
& =3 \times 10^{-36} \mathrm{ergs} \mathrm{cm}{ }^{-3} \mathrm{~s}^{-1} f \epsilon_{\alpha}(1+z)^{9 / 2} .
\end{aligned}
$$

From $\S 3, \mathrm{CMB}$ cooling dominates over recombination and molecular cooling for the conditions of interest. If the ionization fraction is $x$ and the temperature of the ambient medium is $10^{3} T_{\mathrm{amb}, 3} \mathrm{~K}$, the cooling rate is

$$
\Lambda_{\mathrm{CMB}}=1 \times 10^{-39} \mathrm{ergs} \mathrm{cm}^{-3} \mathrm{~s}^{-1} x(1+z)^{7} T_{\mathrm{amb}, 3} .
$$

Equating the two, the temperature is $T_{\mathrm{amb}, 3}=3000 f \epsilon_{\alpha} x^{-1}$ $(1+z)^{-5 / 2}$. If the hydrogen is completely ionized $(x=1)$, $\epsilon_{\alpha}=10$ from Peebles et al. (2000) implies a temperature of $1.5 \times 10^{4} f \mathrm{~K}$ at $z=20$; reducing the ionized fraction raises the temperature. Thus, unless the generation of $\mathrm{Ly} \alpha$ photons occurs almost free of heating $(f \ll 0.01)$, the heating present if this source persisted at moderate redshift would raise the temperature dramatically above what it would be in the standard model, and hence increase the Jeans mass and delay structure formation. The only type of ionization source that could significantly affect the CMB power spectrum and yet not significantly affect structure formation is one with a very steep redshift dependence. If the energy generation rate per volume is $\epsilon \propto(1+z)^{n}$ with $n>7$, heating effects at low redshift will be small. Otherwise, such heating will affect structure formation dramatically.

The richness of data available from upcoming cosmological experiments will rapidly establish whether such ionizing and heating sources exist. If, for example, the first nonlinear baryonic objects did not form until $z \sim 15$ or more recently, evidence of this should be readily apparent in data from many future satellites, including the Next Generation Space Telescope (see, e.g., Robinson \& Silk 2000) and the SIRTF (e.g., Fazio, Eisenhardt, \& Huang 1999), and in the redshifts and absorption-line spectra from distant gamma-ray bursts observed with HETE-2 and Swift (Wijers et al. 1998; Lamb \& Reichart 2000; Blain \& Natarajan 2000). In addition, as more data with better calibrations are available from CMB experiments, the effects at higher $l$ will be more sensitive to the presence of any extra ionization (Miller 2000). In either case, the role of extra ionizing and heating sources in the early universe will be clarified greatly in the next 5 years.

We are grateful to N. Gnedin for useful information and to J. Ostriker, D. Lamb, and A. Loeb for helpful comments on the manuscript. We also thank the referee for suggestions that improved the clarity of the paper. This work was supported in part by NASA grant NAG 5-9756.

\section{REFERENCES}

Ball, G., Narayan, R., \& Quataert, E. 2001, ApJ, 552, 221

Bardeen, J. M., Bond, J. R., Kaiser, N., \& Szalay, A. S. 1986, ApJ, 304, 15 Barkana, R., \& Loeb, A. 2001, Phys. Rep. 349, 125

Blain, A. W., \& Natarajan, P. 2000, MNRAS, 312, L35

Blandford, R. D., \& Begelman, M. C. 1999, MNRAS, 303, L1

Bunn, E. F., \& White, M. 1997, ApJ, 480, 6

Carr, B. J. 1981, MNRAS, 194, 639

Carroll, S. M., Press, W. H., \& Turner, E. L. 1992, ARA\&A, 30, 499
Dalgarno, A., Yan, M., \& Liu, W. 1999, ApJS, 125, 237

de Bernardis, P., et al. 2000, Nature, 404, 955

Fazio, G. G., Eisenhardt, P., \& Huang, J.-S. 1999, Ap\&SS, 269, 541

Gnedin, N. Y. 2000, ApJ, 542, 535

Gnedin, N. Y., \& Ostriker, J. P. 1992, ApJ, 400, 1

Gnedin, N. Y.,, Ostriker, J. P., \& Rees, M. J. 1995, ApJ, 438, 40

Griffiths, L. M., Barbosa, D., \& Liddle, A. R. 1999, MNRAS, 308, 854

Haiman, Z., Abel, T., \& Rees, M. J. 2000, ApJ, 534, 11 
Hanany, S., et al. 2000, ApJ, 545, L5

Heath, D. J. 1977, MNRAS, 179, 351

Hu, W., \& Sugiyama, N. 1996, ApJ, 471, 542

Hummer, D. G. 1994, MNRAS, 268, 109

Lamb, D. Q., \& Reichart, D. E. 2000, ApJ, 536, 1

Lee, A. T., et al. 2001, preprint (astro-ph/0104459)

Lepp, S., \& Shull, J. M. 1984, ApJ, 280, 465

Mather, J. C., Fixen, D. J., Shafer, R. A., Mosier, C., \& Wilkinson, D. T. 1919, ApJ, 512, 511

Miller, M. C. 2000, ApJ, 544, 43

Narayan, R., Mahadevan, R., \& Quataert, E. 1998, in The Theory of Black Hole Accretion Disks, ed. M. A. Abramowicz, G. Björnsson, \& J. E. Pringle (Cambridge: Cambridge Univ. Press), 148

Netterfield, C. B., et al. 2001, ApJ, submitted (astro-ph/0104460)

Ostriker, J. P., \& Gnedin, N. Y. 1996, ApJ, 472, L63

Peebles, P. J. E. 1993, Principles of Physical Cosmology (Princeton: Princeton Univ. Press)
Peebles, P. J. E., Seager, S., \& Hu, W. 2000, ApJ, 539, L1

Pryke, C., Halverson, N. W., Leitch, E. M., Kovac, J., Carlstrom, J. E., Holzapfel, W. L., \& Dragovan, M. 2001, ApJ, submitted (astro-ph/ 0104490)

Puy, D., Alecian, G., Le Bourlot, J., Leorat, J., \& Pineau des Forets, G. 1993, A\&A, 267, 337

Robinson, J., \& Silk, J. 2000, ApJ, 539, 89

Seljak, U., \& Zaldarriaga, M. 1996, ApJ, 469, 437

Silk, J. 1971, A\&A, 12, 421

Tegmark, M., Eisenstein, D. J., Hu, W., \& de Oliveira-Costa, A. 2000, ApJ, 530,133

Tytler, D., O’Meara, J. M., Suzuki, N., \& Lubin, D. 2000, Phys. Scr., T85, 12

Valageas, P., \& Silk, J. 1999, A\&A, 347, 1

Wijers, R. A. M. J., Bloom, J. S., Bagla, J. S., \& Natarajan, P. 1998, MNRAS, 294, L13 